\title{
Product Screening to Multicustomer Preferences: Multiresponse Unreplicated Nested Super-ranking
}

\author{
George J. Besseris ${ }^{1,2}$ \\ ${ }^{1}$ Mechanical Engineering Department, Technological Education Institute of Piraeus, Piraeus, Athens 12201, Greece \\ ${ }^{2}$ Advanced Industrial and Manufacturing Systems, Kingston University, London Surrey KT1 1LQ, UK
}

Correspondence should be addressed to George J. Besseris, besseris@teipir.gr

Received 15 May 2008; Revised 3 September 2008; Accepted 22 October 2008

Recommended by Fugee Tsung

\begin{abstract}
Modern production methods demand the synchronous multicharacteristic optimization of goods. There is a need to diversify a basic product to the importance placed on its individual quality components by a wide spectrum of concerned customers. This work shows how the super-ranking concept may be utilized taking into account relative weights among the implicated responses. The theoretical development is focused on the difficult situation where the optimization is attempted through unreplicated and saturated fractional factorial designs. A nested super-ranking scheme is devised to accommodate a dual weight assignment, first by setting up a single consolidated response per implicated customer and then, in a subsequent step, by incorporating a customer importance rating thus rendering an overall single master response. A demonstration of the proposed method on a pragmatic problem arising in aluminum milling involves optimization due to seven controlling factors concurrently influencing nine product responses modulated by six preference ratings set by a given customer base, respectively. Key benefits of this method are the offered ease of intermixing numerical and categorical data in mainstream multiresponse optimization problems, and keeping customer preferences in perspective through economical, short-cycle screening while relaxing stringent data normality and possible multidistributional effects among the implicated quality characteristics.
\end{abstract}

Copyright (C) 2008 George J. Besseris. This is an open access article distributed under the Creative Commons Attribution License, which permits unrestricted use, distribution, and reproduction in any medium, provided the original work is properly cited.

\section{Introduction}

It is a rare phenomenon that a single dominant product characteristic is the only reason that a product is selected out by inquisitive and well-informed consumers. Consumers are becoming in a blink of an eye aware about new trends and at the same time they shape themselves to new expectations for goods and services they desire. Modern quality culture endorses the hunt for discovering customer expectations that in technical language is converted to merely a host of quality characteristics (or responses). The number of the published work on multiresponse optimization is justifiably growing with no end in sight. There is a multitude of techniques and methods to confront the theoretical and statistical aspects of such difficult problems. However, among the most challenging experimental design schemes, especially in industrial process screenings, are the fractional factorials $[1,2]$. When an expeditious experimental effort is amenable to either a lack of resources or other economic issues, then the unreplication of the scheme is anticipated to be an additional advantage to that end. Furthermore, squeezing out all information from a saturated fractional factorial design gives the experimenter the chance to explore the maximum likely modes that may affect a test product. This practical investigation will make use of such unreplicated and saturated fractional factorial designs to size up statistically product and process parameters that influence many product characteristics. The concept of super-ranking is utilized here for providing objective contrasting among the combined effect rendered by all considered responses concurrently. This last notion is deeply appreciated when designing new processes for new products or improving existing products and it is an everyday concern in mass production. However, a greater problem with immense repercussions in manufacturing is optimizing a product line across a similar workflow that may institute different customer preferences on a given list of product traits. This may be interpreted as optimizing several responses 
simultaneously while taking in account individual response prioritization for each implicated customer concurrently. It is stressed that the individual customer trait hierarchy contributes synchronously to estimating the optimal setting of statistical significant controlling factors. The proposed technique relies on the statistical grounds of nonparametric theory enjoying the well-known benefits rank-ordering statistics offer to cases that demand brief experimentation. One advantage is the inherited exceptional efficiency at small sampling rates. Thus, decision making is achieved by a simple statistical method that competes agreeably with a popular alternative such as the desirability method. Additional advantages discussed for the proposed technique is the convenience in mixing up variable and categorical response data alike. The theory developed here is targeting orthogonal array families members of $\mathrm{L}_{4}\left(2^{3}\right)$, $\mathrm{L}_{8}\left(2^{7}\right), \mathrm{L}_{12}\left(2^{11}\right)$, and $\mathrm{L}_{16}\left(2^{15}\right)$ because of their inherent reliance on low sampling count [3]. A case study drawn from the heavy industry aids in demonstrating the possible usefulness of the tool developed herein. Aluminum canmaking material is modulated by seven controlling factors reflected by the response of nine quality characteristics and indicators weighted each time by a preference priority setting of six customer groups.

\section{Literature Review}

The general theory of design of experiments has been well described in several modern expositions [1-3]. The method of quality loss functions in achieving robust consumer products has been outlined by Clausing and Taguchi [4], Taguchi [5] and Ross [6]. Product multicharacteristic selection has shown to be solvable by fundamental Taguchi methods [7]. Vining and Myers [8] have attempted to bring together the best features of two very popular methods, response surface methodology and Taguchi's robust design in order to produce a superior optimizer hybrid. Along these lines, Tang and $\mathrm{Xu}$ [9] have suggested a unified approach in handling the dual response surface optimization. However, the signal-to-noise ratio concept introduced by Taguchi in optimization problems seems to possess several weaknesses that have received critical attention [10]. There are two indepth lengthy reviews about the current status of robust parameter design that are accompanied with a wealth of references $[11,12]$. However, there are earlier contributions that have introduced the general concept of fitting polynomial regression functions to model parameter selections in multifactor screening [13].

Fractional factorial designs and design of experiment methods remain popular in manufacturing and in mechanical engineering applications [14-18]. Clearly, design of experiments is among the most potent data-mining techniques that may provide quick interpretation to phenomenological behaviors which are much in need at the shop-floor level [19].

The multiresponse optimization problem has been recognized to be a very difficult one. Therefore, there has been a need for insightful direction if it is to reach to meaningful solutions [20]. Competitive business environments do not allow development time for ameliorating product traits on a one-by-one characteristic basis anymore $[21,22]$. It has become particularly important in complex manufacturing environments to navigate through the trivial many responses in order to uncover the indispensable few with respect to the needs of a customer [23]. The first method commending wide acceptance even at present is the involvement of the desirability function in a way that each response is weighted individually while the terminal decision making is derived from a composite desirability measure [24]. Based on this concept, there were several investigations that through selected modifications provided better estimations with respect to the original technique [25]. This method has been extended for predicting operating windows in product designing [26]. The desirability method has become so indispensable to product improvement that is adopted directly in modern quality initiatives such as in Six Sigma for translating and driving towards operational excellence [27]. Ortiz et al. [28] have employed the deterministic prowess of a genetic algorithm to decipher difficult multiresponse behavior by an unconstrained desirability method. It is not a rare event to have several responses that may be related to some degree of correlation among them. In such cases, the method of [29] appears to be a practical outlet. Exponential desirability functions have been developed to counter the cumbersome problem of adjusting the behavior of several mechanical properties for steel at the same time [30]. Kros and Mastrangelo [31] contrasted mixed-type desirability functions in the additive and multiplicative approach with respect to input parameters and response levels. However, no matter the selection of the multiresponse statistical processor, there is insightful information generated by keeping track of the sensitivity analysis results for various ways of solutions [32].

The multiple response surfaces have been treated with the quality loss function concept offering obvious simplicity to the analysis [33]. A compromise approach by Vining [34] has provided promising results. Multiresponse robust design seems to be greatly extended by the method of combined array [35]. Recent research on the area of programming a numerical control machine has turned up useful accounts on multiple comparisons by introducing a new function that incorporates location and dispersion effects [36].

The Taguchi approach continues to find a niche in manufacturing processes even for the case where many characteristics are investigated. Rekindled interest for this method arises because of the convenience this method possesses even for complicated parameter design problems [37]. However, techniques that maintain convincing factor resolution for a multiresponse optimization problem essentially make an attempt to join together the data analysis with a sophisticated decision-making routine. Successful applications of such expert-system-oriented methods have been relied on goal programming [38], neural networks [39, 40], and the utility concept [41]. These methods are solid in their "sieving-through" data capabilities, nevertheless, they may be considered as arduous to the average product developer. 
An alternative approach has been mobilized in reducing the volume of necessary calculations for the multiresponse optimization. When the number of quality characteristics is small, that is two, then the Principal Component Analysis is espoused to reduce the number of responses from two to one so in this case the problem is cast to an unreplicated fractional factorial design solved by the Taguchi method and the pooled analysis of variance (ANOVA) [42]. Tong and Wang [43] have revisited the multiresponse optimization problem and they found that there is significant gain in cohabiting the front-processing response reduction provided by the Principal Component Analysis with the grey relational analysis in the back-end for decision making. All these different ways of attempting to explain the multiresponse optimization issue only prove the incessant necessity for such methods along with the difficulty in encompassing realistic matters to a product developer in a robust design study. Such matters may be an emphasis on economic experimentation, the use of robust statistics at low sampling ranges, the handling of possible intermixing of variables of different nature, that is, numerical and categorical data and so forth. It is expected that there will be enough room for new developments in this critical field that transfixes both areas regarding quality and new technology [44] while at the same time they will become easier to grasp and apply to everyday process improvement projects [45].

The advantages of nonparametric statistics over normal estimators and their inherent versatility and efficiency under any unknown data distribution for short sampling have been collected in the recent work of Conover [46]. It would be an interesting outcome if multiresponse optimization may be elucidated by nonparametric inference methods and this is the subject of the next section.

\section{The Multiresponse Nested super-ranking Approach in Unreplicated Saturated Fractional Factorials}

The possible usefulness of the super-ranking concept in an engineering application that involved unreplicated saturated fractional designs has been demonstrated by Besseris [47, 48]. This concept is further explained in this paper for the case where multiple responses have to be optimized based on a variety of customer priorities placed on the considered responses. A simple theory is built here which borrows elements developed from the super-ranking treatment as previously stated. We contemplate fractional factorial designs of the family series $\mathrm{L}_{4}\left(2^{3}\right), \mathrm{L}_{8}\left(2^{7}\right), \mathrm{L}_{12}\left(2^{11}\right)$, or $\mathrm{L}_{16}\left(2^{15}\right)$ suitable for low sampling screening experimentation. If there are $k$ factors and possibly accompanying interactions, denoted as $X_{1}, X_{2}, \ldots, X_{k}$, then, there are at least $n$ experimental trials that should be performed to relay possibly useful information. For any given arbitrary orthogonal array mentioned above, the corresponding columns of settings may be represented as $X_{1 j}, X_{2 j}, \ldots, X_{k j}(j=1,2, \ldots, n)$. It is sought the concurrent optimization of $m$ distinct, independent responses, denoted as $Y_{1}, Y_{2}, \ldots, Y_{m}$, modulated by these factor settings. Each response then is a vector of dimensionality

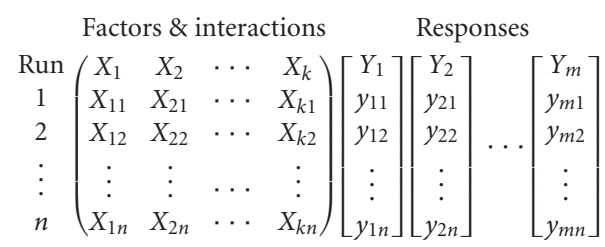

FIGURE 1: Generic representation of an orthogonal array depiction with respect to a group of responses.

$n$, comprising of elements $y_{1 j}, y_{2 j}, \ldots, y_{m j}(j=1,2, \ldots, n)$ (Figure 1).

The super-ranking concept through its squared Euclidean norm formalism as a multicharacteristic measure provides a combined rank-ordered transformation of all implicated responses in a new single manageable master response. Therefore, in a similar fashion to the super-ranking approach, in this work, each response has its elements rank ordered (Figure 2). The rank assignment process follows in the ordinary way, that is, (a) for the case "smaller-the-better," rank one is attributed to the entry possessing the smallest magnitude, rank two for the next smaller entry, and so on; (b) for the "larger-the-better" case, the largest response value gets rank one, the second larger value gets rank two, and so forth. We remark that for the "nominal-the-best" case, it is the absolute value of a response entry from the nominal value that is rank ordered. The rank transformed response elements are simply assigned as $y_{i j} \rightarrow T_{i j}(j=1,2, \ldots, n)$. The next step is to square all response ranked elements.

However, at this point, we introduce the customer preference priority concept which is simply expressed as numerical weight factors, $w_{i}^{l}(i=1,2, \ldots, m$ and $l=1$, $2, \ldots, p)$, assigned after each concerned customer has declared a quality trait hierarchy for the product. For every customer, $l$, out of a total participating cohort of $p$ members, there are as many as $m$ weight factors, which correspond to each response, $i$, of the $m$ considered responses. These weight factors possess the following properties:

$$
\begin{aligned}
& \text { for every } l \quad(l=1,2, \ldots, p): 0 \leq w_{i}^{l} \leq 1 \quad(i=1,2, \ldots, m), \\
& \text { for every } l \quad(l=1,2, \ldots, p): \sum_{i=1}^{m} w_{i}^{l}=1 .
\end{aligned}
$$

Subject to these constraints, the squared Euclidean norm (SEN) measure is still formed just as in the super-ranking formalism but this time each squared-rank element is firstly multiplied by the respective weight factor for a given customer preference priority. This is easily understood from the depiction of Figure 3.

The next step is to form a super ranking (SR) vector by simply rank ordering the $\mathrm{SEN}_{i}^{l}$ elements one more time for each set of customer prioritized weight factors separately. This transformation is better illustrated in Figure 4 .

The $\operatorname{SR}_{i}^{l}(l=1,2, \ldots, p)$ vectors for every customer preference prioritization may be visualized as a set of $p$ 


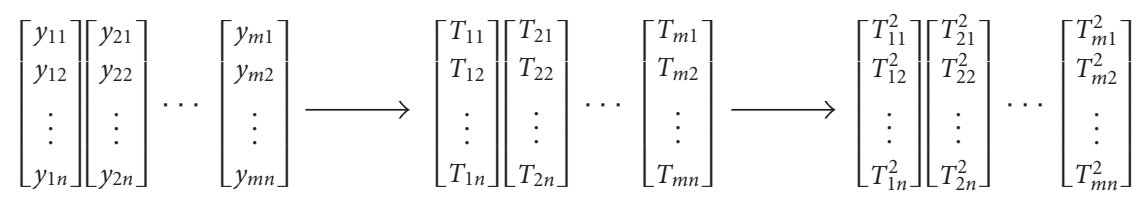

FIGURE 2: Response element rank-order transformation process.

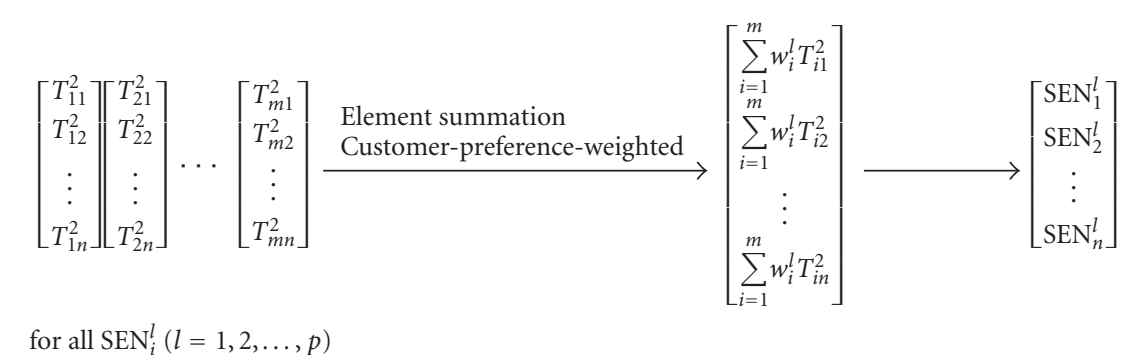

FIGURE 3: Rank consolidation for all response elements to a squared Euclidean norm (SEN).

superranked responses, each containing total weighted response behavior according to the needs of $p$ customers. It is worthwhile to facilitate, in a simplistic manner, the optimal process management of a group of product quality traits synchronously under the various quality response expectations that are reflected by a group of $p$ customers. Assuming all customers are important to this supplier at the same degree then the family of superrank vectors depicted in Figure 4 should be treated concurrently. This problem shapes up to be an application of the super-ranking concept on a group of formed superranked responses. This technique may be suitably called the nested super-ranking approach. Therefore, the nested optimization setup now may be presented in the following form shown in Figure 5.

Repeating now once more the super-ranking technique on the $p$-customer superrank responses by adopting again the squared Euclidean norm, this time denoted as $S_{i}(i=$ $1,2, \ldots, n)$ to distinguish it from the squared Euclidean norm (SEN) which is dedicated to the product characteristics from the previous step. Rank ordering the squared Euclidean norm in the usual manner will result in the master superrank response, $\operatorname{MSR}_{i}(i=1,2, \ldots, n)$, that contains the complete information derived from product quality characteristic performance and customer priority trait setting (Figure 6).

All these manipulations succeed in casting the original problem to that of a single response unreplicated and saturated fractional factorial design lending itself to the treatment outlined in [48]. In Figure 7, it is shown the terminal form of the design. Now, a robust statistical analysis tool should assign the significance of each controlling factor by incurring factor-level contrasts. This is attained by sizing up directly the data grouped for each factor setting separately. This way, factors are ranked according to the smallest statistical significance. The ordinary method to sieve through the effect activeness is to accept a level of significance and reject all those factors that are not meeting or exceeding the predefined level of significance. The nonparametric test of Wilcoxon-Mann-Whitney or rank-sum test is selected for the analysis because of its high efficiency and its nondependency on statistical distribution functions.

To enable the use of Wilcoxon-Mann-Whitney test for data collected by a 2-level orthogonal array, such as the $\mathrm{L}_{8}\left(2^{7}\right)$ highlighted in the case study, response entries accrued in the master superrank column of Figure 7 should be separated in two groups according to the factor level they reflect. The case study that follows this discussion will assist in comprehending this type of grouping. Then, what remains to be done is either to sum the elements that belong to either of the two-factor settings and compare it with a critical value tabulated for several levels of significance in the appendix of an elementary statistics textbook, or feed the two created sample groups into a software package, thus obtaining the statistical significance of their medians. Since the level of computational complexity for nested superranking calculations is increasingly more demanding than that of a simple super-ranking application, the mere use of a calculator may prove to be cumbersome in this case. Therefore, the use of an ordinary spreadsheet will suffice in order to carry out the necessary comparisons without the need to resorting to professional statistical software packages. This may assist engineers to assimilate easier the method who, otherwise, do not feel comfortable with such statistical software products.

The hypothesis testing for this kind of data comparisons reflects the examination of the predicament that the two formed samples belong in the same common population for all MSR entries, for each considered factor individually. Since we defined $X_{1}, X_{2}, \ldots, X_{k}$ the factors (including possible interactions) that need to be examined, then $X_{1}^{1}$ and $X_{1}^{2}, X_{2}^{1}$ and $X_{2}^{2}, \ldots, X_{k}^{1}$ and $X_{k}^{2}$ are their respective medians of their setting outcomes which correspond to levels 1 and 2 of a presumed 2-level orthogonal array. As expected, $k$ is equal to seven for the $\mathrm{L}_{8}\left(2^{7}\right) \mathrm{OA}$ showcased in the next section. Then, 


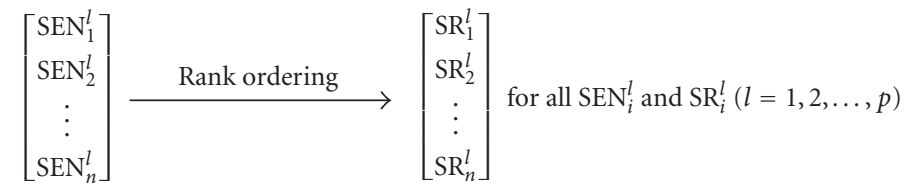

FIGURE 4: Rank ordering of SEN data to SR vector form per customer, $l$.

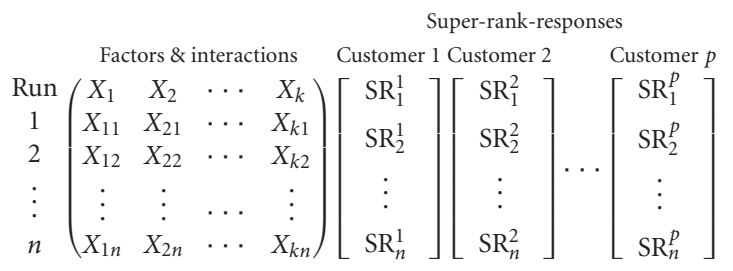

FIGURE 5: Representation of an orthogonal array against a number of $p$ superrank responses.

for each factor the null hypothesis, $\mathrm{Ho}$ and its alternative $\mathrm{H}_{1}$, will be

$$
\begin{aligned}
& \text { HO: } \text { Median } X_{1}^{1}=\text { Median } X_{1}^{2} \\
& \quad \text { OR } \mathbf{H}_{1} \text { : Median } X_{1}^{1} \neq \text { Median } X_{1}^{2}, \\
& \text { HO: } \text { Median } X_{2}^{1}=\text { Median } X_{2}^{2} \\
& \quad \text { OR } \mathbf{H}_{\mathbf{1}} \text { : Median } X_{2}^{1} \neq \text { Median } X_{2}^{2}, \\
& \vdots \\
& \text { HO: } \text { Median } X_{k}^{1}=\text { Median } X_{k}^{2} \\
& \quad \text { OR } \mathbf{H}_{1} \text { : Median } X_{k}^{1} \neq \text { Median } X_{k}^{2},
\end{aligned}
$$

where $k$ is equal to seven for the case of an $\mathrm{L}_{8}$ orthogonal array.

Customarily, the statistic that needs to be computed is the smaller sum of ranks when all observations from each setting separately are pooled into one group while keeping the tag on for each sum of ranks for each setting. If a critical value defined for a specific level of significance for the Wilcoxon-Mann-Whitney is exceeded then the null hypothesis is accepted. Otherwise, the alternative hypothesis is accepted.

\section{A Case Study}

An important industry is aluminum products manufacturing and great interest receives the aluminum-alloy canbody stock which is indispensable material for seamless onepiece containers in the beverages and soft drinks sectors. The continuous attention to this product is manifested by the enormous amount of patented work produced every year necessitated by ever economical and lighter containers which possess improved strength properties in line with better machinability during rolling processing and afterwards "drawing and ironing" forming operations. The case study presented here will focus on the strip production of such material that involves several phases including aluminum ingot casting, heat-treatment homogenization, hot rolling, anneal treatment, and cold rolling to the desired gauge specified by a can maker. For this specific study, nine responses will be attempted to be optimized synchronously to seven selected process and product factors. The nine responses regard alloy AA3004 and they are as follows: (1) tensile yield strength (TYS); (2) tensile ultimate strength (TUS); (3) tensile total elongation (TTE); (4) earing (Ear); (5) surface quality (SQ); (6) necking/flange quality (N/F); (7) terminal thickness gauge (Th); (8) terminal width gauge (W); and (9) buckle strength (BS). The definitions for the first three responses are those found in standard material science terminology. TYS and TUS are measured in kpsi unit, and TTE is measured in terms of percent deformation. TYS and TUS are optimized about their respective target values with further supplemented lower and upper bounds usually set by the customer. The TTE is expected to be maximized for improved formability while there is desirable minimum value which below that the material is regarded as a failure. Earing occurs after the two-phase process of deep drawn containers stamped from aluminum textured sheet in the cupping press is further subjected to ironing in a bodymaker to achieve the specified can height. This property essentially quantifies the difference between the ears (hills) and the troughs (valleys) appearing at the edge of a can. Earing is also measured in terms of a percentage and since it is undesirable because it interferes with flange width consistency during the lid fitting process, it needs to be minimized. Thickness and width are tabulated in terms of a difference for each of them reflecting the extent of the discrepancy; the measured gauge of examined samples possess with respect to can-maker's specified nominal values, respectively. Coincidentally, this form of representation serves two equally important purposes. First, measurements do not need to be manipulated according to a "nominal-isbest" treatment. Taking simply the difference of each data point to the nominal value, the condition is transformed, in the "super-ranking" formulation, to the "smaller-isbetter" optimization category. Thus, what are sought are the optimum conditions that will drive this difference to zero. Second, the identity of the can maker or a group of them as it may be is not revealed through this study since the combination of terminal strip thickness and width specifications usually relates to particular can manufacturing enterprises. Surface quality is categorical variable in ordinal scale based on alloy surface crystallographic texture and it is rendered after a metallurgical laboratory examination. The rating ranges from a scale 1 to 5. "Very good" is reflected by rating 5, "good," "average," "poor," and "very poor" are set aside for ratings $4,3,2$, and 1 , respectively. "Necking and 


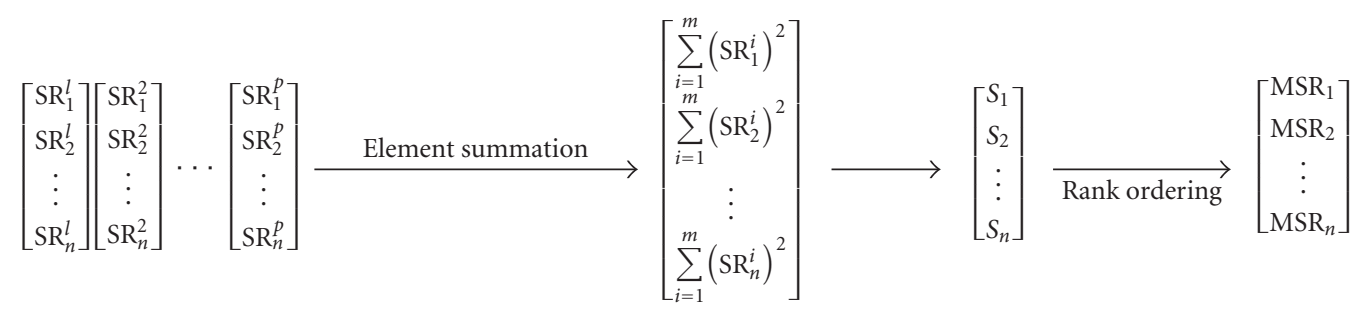

FIgURE 6: Rank consolidation for all response elements to superranked form.

Factors \& interactions Master-super-rank

$\operatorname{Run}$
1
2
$\vdots$
$n$$\left(\begin{array}{cccc}X_{1} & X_{2} & \cdots & X_{k} \\ X_{11} & X_{21} & \cdots & X_{k 1} \\ X_{12} & X_{22} & \cdots & X_{k 2} \\ \vdots & \vdots & \cdots & \vdots \\ X_{1 n} & X_{2 n} & \cdots & X_{k n}\end{array}\right) \quad\left[\begin{array}{c}\mathrm{MSR}_{1} \\ \mathrm{MSR}_{2} \\ \vdots \\ \mathrm{MSR}_{n}\end{array}\right]$

FIGURE 7: Representation of an orthogonal array against $p$ number of superrank responses.

flange" characteristic is a quality property that is evaluated in the customer's can-making systems. It has to do with the consistency the material has in shaped can form after drawing and ironing. Flange width is crucial in fitting the lid to the necked can at the specifications dictated by lid dimensions. Just as with the surface quality characteristic the measuring scale for $\mathrm{N} / \mathrm{F}$ is of the same nature, the only difference being that is an evaluation that it is provided by the customer. Buckle strength (dome reversal pressure) is measured in psi. It is considered as an added material strength property that dome-shaped can bottoms should possess to withstand canned internal pressurized contents. This restriction is communicated by the customer in terms of a minimum specification pressure value which is the point that the can bottom inverts its shape from concave downward to convex downward. Therefore, this characteristic should be maximized.

A selected group of seven factors modulates the responses outlined above. Two of these are the manganese (Mn) and magnesium (Mg) contents expressed as a percentage by weight. Homogenization treatment was studied according to two process parameters which are the furnace temperature (TempH) and duration of slab heating in the furnace (TimeH). Moreover, the importance of two annealing treatments was investigated, one after hot rolling and the other after cold reduction. In both cases, the process parameter was the annealing temperature (in ${ }^{\circ} \mathrm{C}$ ) as the annealing interval was maintained constant all through the experiments. The temperature for the first annealing treatment is coded as AnA and for the second treatment as AnB. The last factor is the cold reduction (CR) referring to the thickness gauge reduction incurred by cold rolling and it is recorded in terms of a percentage with respect to the incoming workpiece thickness gauge. The study is oriented towards a screening phase investigation. Therefore, the management team at this point would prefer to observe comparative behavior among all engaging factors at some reasonable process and product parameter extremities that nevertheless are not foreign to tested recipes in previous patent work. Hence, it was thought that a two-level $\mathrm{L}_{8}\left(2^{7}\right)$ orthogonal array should be utilized in selecting the appropriate trial run combinations. Resorting to OAs makes sense from an economical perspective in this situation because each workpiece resulting to final product may pile up a cost of the order of $\$ 100000$ requiring a combined cycle time of one to two months to produce it. By combined cycle time, it is meant time-to-product-completion in both sites, at producer's and customer's, respectively. The comprehensive input selections are tabulated in Table 1.

Data gathered in Table 2 is given in the form of the $\mathrm{L}_{8}\left(2^{7}\right) \mathrm{OA}$ arrangement to enhance readability. In Table 3, response data received a rank ordering for each response separately where the event of a tie is honored. Responses of TYS and TUS are ranked according to their differences from the targeted values of 46 and $49 \mathrm{kpsi}$, respectively. The smaller the absolute difference, the lower the rank each response entry receives. Similarly, the response gauge differences for Th and $\mathrm{W}$ are ranked based on their absolute differences with their nominal values. It is interesting to observe the tie occurrence for the categorical ordinal data for responses SQ and $\mathrm{N} / \mathrm{F}$ when translated to the corresponding rank variable. There is reverse meaning in these values as the maximum for the ordinal scale of 5 will get the first rank, the rating of 4 will be rank rated as 2 and so forth. However, it is noteworthy to stay at the convenience this method may offer in mingling categorical data with numerical data of any dimensional basis in any multiresponse optimization circumstances. The rank ordering provides uniform scaling to all measures thus simplifying enormously the data analysis in the subsequent step. The coded names for the transformed rank-ordered responses mirror those of the original data response variables with the prefix addition of "R," for "ranked," as they appear in Table 3.

At this point, it would be interesting to discover the factors that may prove to be statistically important in influencing all responses concurrently taking in account the various ratings that may be placed on each of the implicated responses by a host of can makers (customers) simultaneously. This very realistic problem causes cumbersome alterations in production schedules of this nature. Extensions of this notion to other massively produced goods are easily perceived. From the standpoint of the impending statistical analysis, this calls for what has been described 
TABLE 1: Factor coding and factor settings for the multiresponse optimization study.

\begin{tabular}{lccc}
\hline Coded symbols & Control factor & Level 1 & Level 2 \\
\hline $\mathrm{Mn}$ & Manganese content $(\%)$ & 0.6 & 1.5 \\
$\mathrm{Mg}$ & Magnesium content $(\%)$ & 0.7 & 1.8 \\
Temph & Homogenization temperature $\left({ }^{\circ} \mathrm{C}\right)$ & 530 & 580 \\
Timeh & Homogenization duration (hours) & 6 & 24 \\
$\mathrm{AnA}$ & Annealing before cold rolling $\left({ }^{\circ} \mathrm{C}\right)$ & 260 & 370 \\
$\mathrm{AnB}$ & Annealing after cold rolling $\left({ }^{\circ} \mathrm{C}\right)$ & 390 & 560 \\
$\mathrm{CR}$ & Cold reduction $(\%)$ & 35 & 65 \\
\hline
\end{tabular}

TABLE 2: Data collected for the nine responses due to the adjustments of the seven selected factors stated in Table 1 in terms of a $\mathrm{L}_{8}\left(2^{7}\right) \mathrm{OA}$ arrangement.

\begin{tabular}{cccccccccccccccc}
\hline Mn & Mg & TempH & TimeH & AnnA & AnnB & CR & TYS & TUS & TTE & Ear & SQ & N/F & Th & W & BS \\
\hline 1 & 1 & 1 & 1 & 1 & 1 & 1 & 38 & 41 & 2.0 & 2.3 & 4 & 3 & 1 & 1 & 92 \\
1 & 1 & 1 & 2 & 2 & 2 & 2 & 51 & 53 & 2.3 & 2.7 & 5 & 1 & 0 & 2 & 96 \\
1 & 2 & 2 & 1 & 1 & 2 & 2 & 54 & 56 & 3.0 & 2.9 & 3 & 3 & 0 & 2 & 99 \\
1 & 2 & 2 & 2 & 2 & 1 & 1 & 45 & 48 & 3.2 & 3.1 & 3 & 2 & 1 & 1 & 95 \\
2 & 1 & 2 & 1 & 2 & 1 & 2 & 44 & 47 & 1.7 & 1.8 & 5 & 3 & -1 & 0 & 91 \\
2 & 1 & 2 & 2 & 1 & 2 & 1 & 43 & 45 & 2.7 & 2.8 & 2 & 4 & 0 & 1 & 92 \\
2 & 2 & 1 & 1 & 2 & 2 & 1 & 50 & 51 & 4.0 & 3.6 & 3 & 3 & -1 & 2 & 94 \\
2 & 2 & 1 & 2 & 1 & 1 & 2 & 47 & 50 & 2.0 & 2.2 & 2 & 5 & 0 & 1 & 93 \\
\hline
\end{tabular}

in the methodology section of this article as nested superranking in unreplicated and saturated fractional factorial design. Indeed, application of the super-ranking method on weighted responses according to each participating customer preferences will generate a single superranked response for each of the customers separately. Pooling all individual customers superranked responses to a new single master superranked response, this time across all customer base considered and performing the super-ranking methodology one more time on this single response, it would generate a solution that may be worth of interpretation.

Before proceeding with the computations, it would be beneficial to check the results shown in Table 3 with some other competing scheme. This test would provide confidence in handling such complex problem as outlined in the previous paragraph. To this author knowledge, it was not easy to retrieve other well-tested and easily accessed method on multiresponse optimization worked out in concert with orthogonal arrays besides the highly regarded technique of desirability analysis. Since SQ and N/F responses are assigned categorical data and the "Response Optimizer" tool in MINITAB 15 does not discriminate against continuous or discrete data, we treated the data shown on Table 3 unaltered. The input bounds for the MINITAB analysis are given in Table 4 and they are these that are specified by the average customer. To keep things simple at this stage there was no attempt to introduce importance ratings or weights, according to the terminology followed in the desirability analysis. It was thought that this may provide a better sense of comparison between the two methods. The output produced by MINITAB 15 is tabulated in Table 5. Clearly, the overall optimization effort may be characterized as not a successful one as it may be inferred by the composite desirability of about 0.43 . Nevertheless, at this level of difficulty, the desirability analysis suggests that the responses TYS, TUS, and $\mathrm{W}$ have been predicted in a satisfactory fashion with the Th being exceptional.

Furthermore, DA forces a solution for Mg and AnA that do not involve one of the two investigated factor levels. For viewing convenience, the optimized response graphs produced by MINITAB 15 are shown in Figures 8(a) and 8 (b). It should be noted that the solution is portrayed in two parts since the long list of responses could not be fitted in one view as the generated solution appears in a roll-down window form hiding some of the responses. In turn, this causes duplication of some of the response results between the two graphs. However, as it can be witnessed from Figure 8 by focusing on the subplots for composite desirability, it may be inferred that factors $\mathrm{TempH}$, TimeH, and AnA definitely may be omitted from further consideration.

The corresponding solution obtained through the original super-ranking method is presented in Table 6. The Mann-Whitney test assigns statistical significance to median central tendencies between factor levels. It is found that only the homogenization duration rejects the null hypothesis at a significance level of 0.05 . This seems logical as for level 1 and 2, the median SSRs are 253.3 and 201.6, respectively. Thus, in contrast to the result provided by the desirability analysis method, level 2 should be the optimal setting according to super-ranking. The next three more important factors that happen to possess the same statistical significance of 0.235 are Mn, Temph, and AnB. This conclusion is in 
TABLE 3: Rank ordering of data presented in Table 2.

\begin{tabular}{|c|c|c|c|c|c|c|c|c|c|c|c|c|c|c|c|c|c|}
\hline $\mathrm{Mn}$ & Mg & TempH & TimeH & AnnA & AnnB & CR & RTYS & RTUS & RTTE & REar & RSQ & $\mathrm{RN} / \mathrm{F}$ & RTh & RW & RBS & SSR & SR \\
\hline 1 & 1 & 1 & 1 & 1 & 1 & 1 & 7.5 & 8.0 & 6 & 3 & 3.0 & 4.5 & 6.5 & 3.5 & 6.5 & 291.25 & 8 \\
\hline 1 & 1 & 1 & 2 & 2 & 2 & 2 & 6.0 & 5.5 & 5 & 4 & 1.5 & 8.0 & 2.5 & 7.0 & 2.0 & 232.75 & 5 \\
\hline 1 & 2 & 2 & 1 & 1 & 2 & 2 & 7.5 & 7.0 & 3 & 6 & 5.0 & 4.5 & 2.5 & 7.0 & 1.0 & 251.75 & 6 \\
\hline 1 & 2 & 2 & 2 & 2 & 1 & 1 & 1.5 & 1.5 & 2 & 7 & 5.0 & 7.0 & 6.5 & 3.5 & 3.0 & 195.00 & 2 \\
\hline 2 & 1 & 2 & 1 & 2 & 1 & 2 & 3.0 & 3.5 & 8 & 1 & 1.5 & 4.5 & 6.5 & 1.0 & 8.0 & 216.00 & 4 \\
\hline 2 & 1 & 2 & 2 & 1 & 2 & 1 & 4.0 & 5.5 & 4 & 5 & 7.5 & 2.0 & 2.5 & 3.5 & 6.5 & 208.25 & 3 \\
\hline 2 & 2 & 1 & 1 & 2 & 2 & 1 & 5.0 & 3.5 & 1 & 8 & 5.0 & 4.5 & 6.5 & 7.0 & 4.0 & 254.75 & 7 \\
\hline 2 & 2 & 1 & 2 & 1 & 1 & 2 & 1.5 & 1.5 & 7 & 2 & 7.5 & 1.0 & 2.5 & 3.5 & 5.0 & 158.25 & 1 \\
\hline
\end{tabular}

TABLE 4: Required response bound inputs for MINITAB 15 data analysis by the desirability method.

\begin{tabular}{|c|c|c|c|c|}
\hline Response & Lower & Target & Upper & Optimization goal \\
\hline TYS & 40 & 46 & 52 & Target \\
\hline TUS & 43 & 49 & 55 & Target \\
\hline TTE & 1.5 & 3 & & Maximization \\
\hline Ear & & 0 & 2 & Minimization \\
\hline SQ & 3 & 5 & & Maximization \\
\hline $\mathrm{N} / \mathrm{F}$ & 3 & 5 & & Maximization \\
\hline Th & & 0 & 1 & Minimization \\
\hline W & & 0 & 1 & Minimization \\
\hline BS & 90 & 95 & & Maximization \\
\hline
\end{tabular}

TABLE 5: Optimization by the desirability method.

\begin{tabular}{l}
\hline \multicolumn{1}{c}{ Response optimization } \\
\hline $\mathrm{Mn}=2$ \\
$\mathrm{Mg}=1.35$ \\
$\mathrm{TempH}=2$ \\
$\mathrm{TimeH}=1$ \\
$\mathrm{AnA}=1.48$ \\
AnB $=1$ \\
$\mathrm{CR}=2$ \\
\hline \multicolumn{1}{c}{ Predicted responses } \\
\hline $\mathrm{TYS}=44.73$, desirability $=0.789$ \\
$\mathrm{TUS}=47.77$, desirability $=0.796$ \\
$\mathrm{TTE}=1.80$, desirability $=0.206$ \\
Ear $=1.88$, desirability $=0.056$ \\
$\mathrm{SQ}=3.91$, desirability $=0.457$ \\
$\mathrm{~N} / \mathrm{F}=3.94$, desirability $=0.474$ \\
Th $=-0.74$, desirability $=1.000$ \\
$\mathrm{~W}=0.17$, desirability $=0.823$ \\
$\mathrm{BS}=91.88$, desirability $=0.376$ \\
\hline Composite desirability $=0.429$
\end{tabular}

conflict with that reached by the desirability method in the previous paragraph. One more attempt in resolving this difficult problem is to accept the sum of squared individual ranks, that is, the squared Euclidean norm according to [48], as a single response in Table 3. This response is
TABLE 6: Statistical significance assignment according to the superranking concept.

\begin{tabular}{cclc}
\hline Levels & Median ranks & $\begin{array}{l}\text { Mann-Whitney } \\
\text { test statistic W }\end{array}$ & $P$ \\
\hline Mn1 & 5.5 & 21 & .235 \\
Mn2 & 3.5 & & \\
Mg1 & 4.5 & 20 & .332 \\
Mg2 & 4.0 & & .235 \\
Temph1 & 6.0 & 21 & \\
Temph2 & 3.5 & & .030 \\
Timeh1 & 6.5 & 25 & .500 \\
Timeh2 & 2.5 & & \\
AnA1 & 4.5 & 18 & .235 \\
AnA2 & 4.5 & & .332 \\
AnB1 & 3.0 & 15 & \\
AnB2 & 5.5 & & \\
CR1 & 5.0 & 20 & \\
CR2 & 4.5 & & \\
\hline
\end{tabular}

listed as SSR in the same table. Therefore, the popular tests for single unreplicated and saturated response offered by the half-normal method and the Pareto analysis/Lenth test may be enforced here. This action is partly justifiable because as it can be seen from Table 11, the normality of all individual factor levels is found to be reasonable, according to Anderson-Darling test, just as it is for the total eight points in the SSR column as demonstrated in Figure 11(a).

However, the output from MINITAB 15 in Figure 9 (plots (a) and (b)) produces admittedly no strong candidate even at the significance level of 0.10 . These results advocate further the difficulty of handling convincingly these kinds of multiresponse problems thus justifying the continuous need for advanced probing techniques in the area of multicharacteristic optimization.

At this point, let us return to the initial problem of detecting possible factors that influence the total superranked behavior no matter what the customer prescription for the response weights it may be. For illustrational purposes, this paper will consider the optimization problem based on the importance rating given by six can makers. The relative weights corresponding to each of the customers are summed total to unity while they are applied on the squared ranks for 


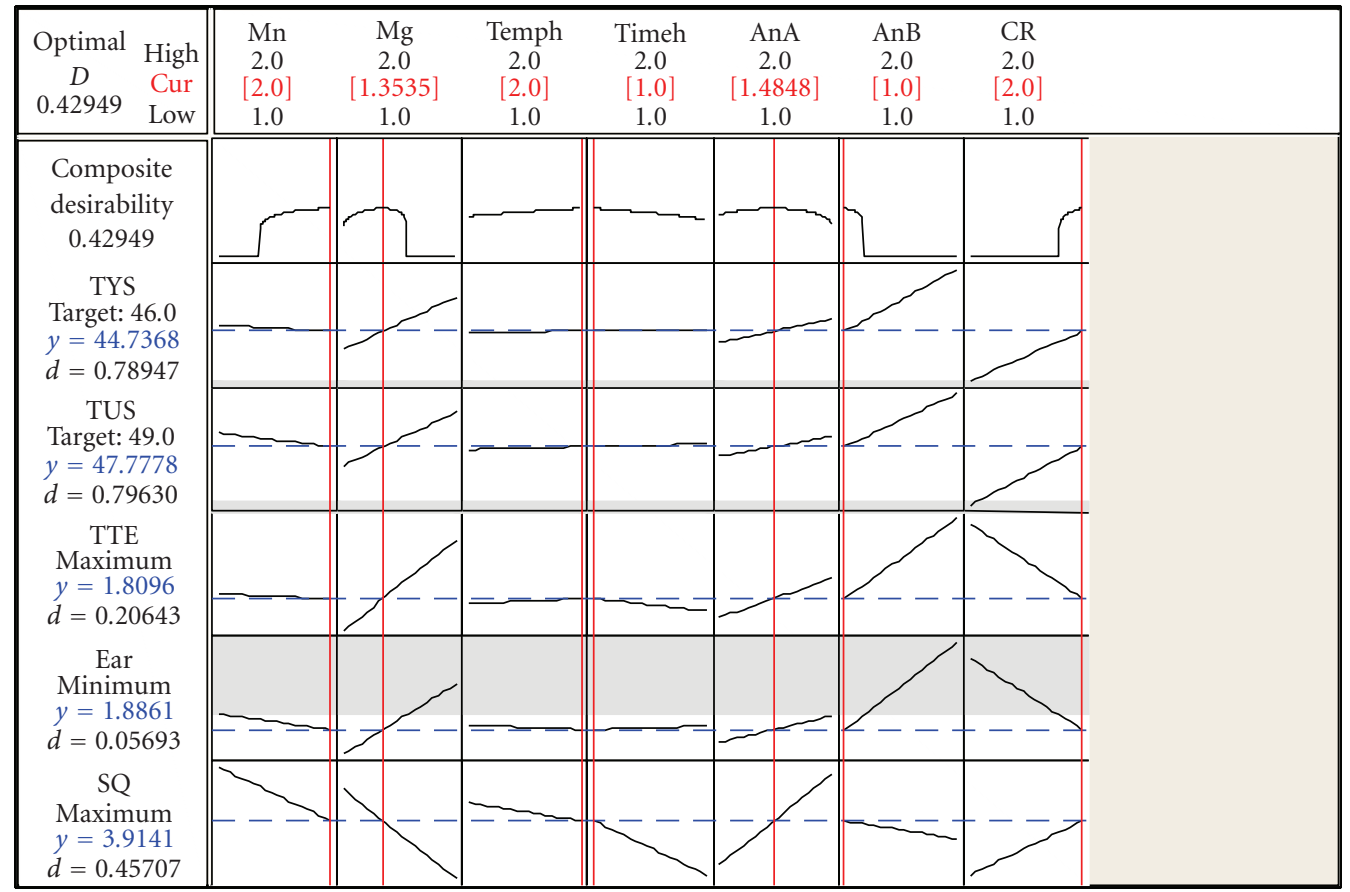

(a)

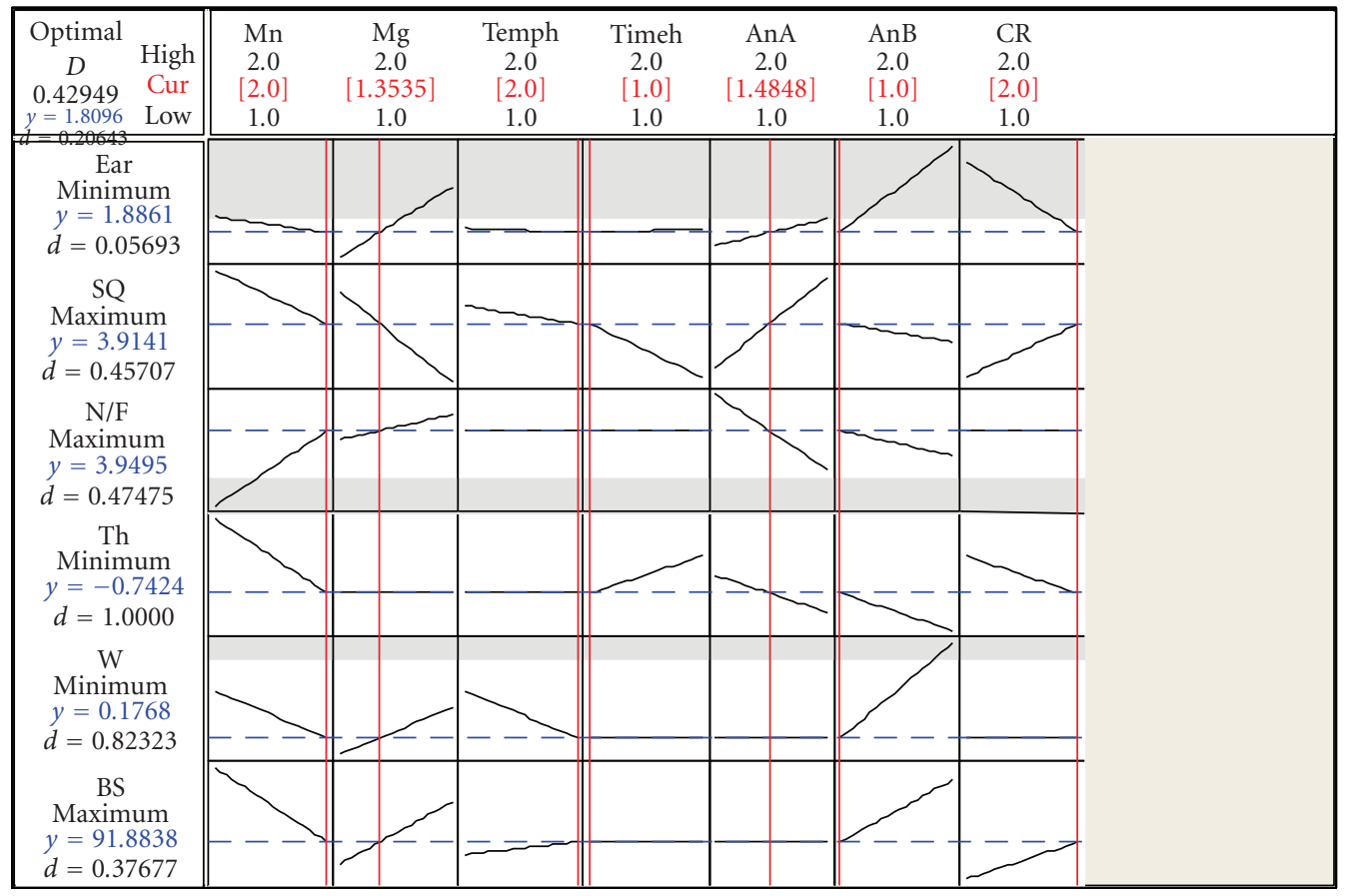

(b)

Figure 8: Graphical solution for the nine responses for the no-weights, no-importance-rating optimization problem based on the data of Table 2 (plots (a) and (b) offer the complete solution according to the desirability method (MINITAB 15)).

each response separately. Therefore, for the six can makers there will be six individual superrank responses. All these responses will face one more level of squaring and addition thus creating a new squared Euclidean norm which a final ranking will generate a master superrank column to be contrasted for factor importance according to the original $\mathrm{L}_{8}\left(2^{7}\right) \mathrm{OA}$. The selected input weights for the six can makers are given at Table 7. Table 10 lists the super-ranking response for each of the six can makers separately. The responses are coded according to the customer identification, for example, 


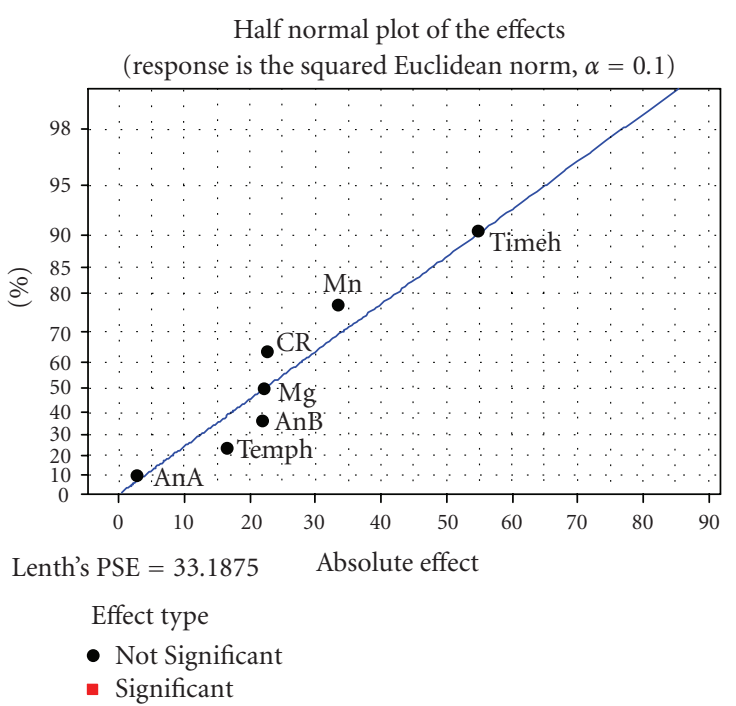

(a)

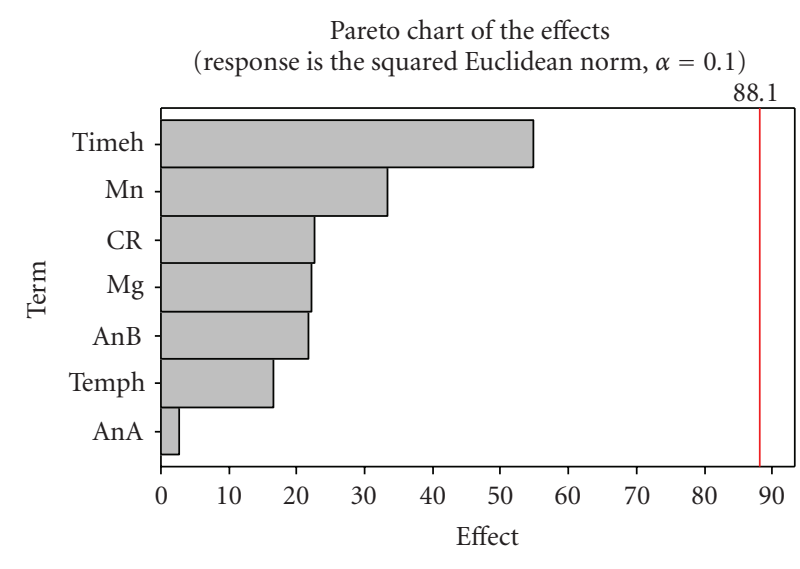

Lenth's PSE $=33.1875$

FIgURE 9: Data for SSR from Table 3 tested with (a) the half-normal test and (b) Pareto analysis/Lenth test.

the super-ranking response created by the weights set out by can maker A is listed as SRA, for can maker B as SRB and so forth. On the same table, it is also listed the combined superranking variable, MSR, the acronyms standing for "master superrank" response, which contains all modulations in the nine responses due to the six can makers. Therefore, this quantity may guide the discovery of important controlling factors that would influence simultaneously all considered responses for all participating customers. The MSR is created by squaring the individual ranks for every customer superranking response on Table 10, that is, by creating a new Euclidean norm that encompasses the customer dimensionality across all trial run selections of the $\mathrm{L}_{8}\left(2^{7}\right) \mathrm{OA}$. Thus, this essentially justifies the need for a nested super-ranking routine. This value is listed as MSSR, for master sum of squared ranks, in Table 9. For convenience, in the same table, it also is listed the sum of squared ranks for every individual can maker. The coding for these values is attributed for each can maker individually, that is, for can maker A, the SSRA labeling on Table 9 stands for sum of squared ranks for can maker A, SSRB for can maker B and so forth. By ranking the elements of MSSR column (Table 9), the rank-ordering outcomes for MSR are generated at Table 10. Treating the MSR entries with the classical method of Wilcoxon-MannWhitney, just as it was discussed in Besseris [48], then the statistical significance of the consolidated behavior of all nine responses due to preferential selection of six can makers owing to the seven controlling factors is tabulated in Table 8. The first impression from this analysis is that the homogenization duration is statistical important at a significance level of 0.10 while is very close to solidify this indication at a significance level of 0.05 . Moreover, it is seen that the factor level Timeh2 amasses the smallest median of the two levels which corresponds to a setting (from Table 1) of 24 hours. At this stage, there are no other controlling factors that may provide statistically important influence on the combined behavior of all implicated responses simultaneously for the traditionally accepted significance level settings of 0.05 and 0.10 . For practical reasons, while understanding the enhanced complexity of this optimization problem at hand, one may desire to consider more factors for setting adjustment. If someone is willing to accept the controlling influence of factors $\mathrm{Mn}$ and $\mathrm{AnB}$ at significance level of 0.156 , then the desired factor settings should be $\mathrm{Mn} 2$ and AnB1 which physically correspond to a manganese content of $1.5 \%$ and an annealing temperature treatment after the cold reduction of $390^{\circ} \mathrm{C}$.

It is noteworthy to mention that the behavior of the super-ranking response for the case where no specific information for the importance rating provided by the customers due to the controlling factors (Table 6) is essentially the same as that for super-ranking response modulated by the response priorities of the group of the can makers in a statistical sense. However, the statistical significance for the three considered factors above, Timeh, Mn, and AnB are pointing towards different direction. In other words, the inclusion of customer multipreferences caused a weakening of the statistical significance of Timeh from 0.030 to 0.056 , while for $\mathrm{Mn}$ and $\mathrm{AnB}$ contributed an observed increase in statistical significance from 0.235 for both of them to 0.156 for each of the two, respectively. Overall, the same conclusion would be reached qualitatively for establishing the important controlling factors if we had used the super-ranking information collected under the heading SR at Table 3 (or Table 10). This means that for the specific group of response priorities assigned to the customer base of Table 7 the modulation of individual rank responses were not strong enough to alter the behavior of the composite response ranking. This is quite surprising if one takes in account how different the relative weights were as nominated by the six can makers in Table 7. Obviously, it is a coincidental occurrence attached only the particular combination of weights and can makers. 
TABLE 7: The nine response weights as listed by the six participating can makers.

\begin{tabular}{lccccccccccc}
\hline CUSTOMER & TYS & TUS & TTE & Ear & SQ & N/F & Th & W & BS & TOTAL \\
\hline A & 0.15 & 0.15 & 0.15 & 0.2 & 0.1 & 0.1 & 0.05 & 0.05 & 0.05 & 1 \\
B & 0.12 & 0.12 & 0.11 & 0.2 & 0.15 & 0.1 & 0.05 & 0.05 & 0.1 & 1 \\
C & 0.3 & 0.3 & 0.3 & 0.02 & 0.02 & 0.01 & 0.01 & 0.02 & 0.02 & 1 \\
D & 0.1 & 0.1 & 0.1 & 0.2 & 0.1 & 0.05 & 0.05 & 0.15 & 0.15 & 1 \\
E & 0.2 & 0.2 & 0.2 & 0.3 & 0.02 & 0.02 & 0.02 & 0.02 & 0.02 & 1 \\
F & 0.05 & 0.05 & 0.1 & 0.1 & 0.05 & 0.05 & 0.3 & 0.2 & 0.1 & 1 \\
\hline
\end{tabular}

TABLE 8: Statistical significance assignment according to the superranking concept for simultaneous six-customer optimization.

\begin{tabular}{lclc}
\hline Levels & Median ranks & $\begin{array}{l}\text { Mann-Whitney } \\
\text { Test Statistic W }\end{array}$ & $P$ \\
\hline Mn1 & 6.0 & 22 & .156 \\
Mn2 & 3.5 & & .232 \\
Mg1 & 4.5 & 21 & \\
Mg2 & 3.5 & & .332 \\
Temph1 & 5.5 & 20 & \\
Temph2 & 3.5 & & .056 \\
Timeh1 & 6.5 & 24 & .332 \\
Timeh2 & 3.0 & & .156 \\
AnA1 & 5.5 & 20 & \\
AnA2 & 4.0 & & .332 \\
AnB1 & 2.5 & 14 & \\
AnB2 & 5.5 & & \\
CR1 & 5.0 & 20 & \\
CR2 & 4.0 & & \\
\hline
\end{tabular}

It is stressed that in order to form the ranked entries for the columns SRA, SRB, and so forth, at Table 10, first the weighted-squared-rank-sum (squared Euclidean norm) to customer priorities for each can maker is formed at Table 9, expressed under the headings SSRA, SSRB, and so forth.

Two alternative ways were used to estimate the effect of the seven controlling factors on the combined weightedsquared-rank-sum response or the master sum of squared ranks response, MSS (Table 9). First, we observe from the Anderson-Darling normality analysis of the fourteen-factor settings that only two of them seem to have questionable indication at a significance level of 0.10 , that is, $\mathrm{Mg}_{1}$ and $\mathrm{AnB}_{1}$ (Table 12). Furthermore, the distribution of the eight elements of response MSS seems to comply with normality (Figure 11(b)). Therefore, we apply two popular tests for unreplicated and saturated designs: (a) the half-normal test and (b) the Pareto analysis/Lenth test by making use the respective module in MINITAB 15. The results are shown in Figure 10 as plots $\mathrm{a}$ and $\mathrm{b}$ for the two corresponding graphical tests. It is clear that both tests are not able to decipher any dominating contributions from the seven parameters that we examined. Indeed, likely dependencies on the master sum of weighted squared ranks (MSS) are remote even at the level of significance of 0.10 . In spite of no meaningful active effects, the Pareto analysis/Lenth test has the parameter
Timeh leading the rest of them. On the other side, the half-normal test cannot distinguish a factor nomination at all for this particular case study. At this point, no further comparisons may be made between the nested super-ranking method and the two classical graphical methods employed here. However, possible reasons for these severe discrepancies may be partially explained by the discussion in Besseris [47]. Hopefully, this irreconcilable condition on predicted results between well-known graphical means and robust permutation-based tests may become ground for additional investigation in forthcoming publications.

\section{Discussion}

In this work, there was an attempt to interpret synchronous multiresponse optimization information under the added constraint of weighted modulation imposed on the individual influence for each of the engaging responses. To this end, a simple statistical model has been developed based on the rank-ordering concept propounded by Wilcoxon, and Mann and Whitney (WMW). This weighted-response model accumulates the rank-ordered information for each response in linear fashion and creates a new superrank response that is processed by the WMW nonparametric test. Therefore, a terminal single response contains compressed information from a given number of quality characteristics while representing all of them with a preassigned level of importance among them. Mainstream competing techniques that incorporate response weighting in their scheme mainly spawn around the concept of either the desirability analysis or some indigenous expert system. Both of these methods quite often require a professional software package to assist in the decision-making and it is handled meaningfully by a well-trained specialist. Furthermore, mixing up expert systems with statistical techniques will always relay useful, well-received conclusions. However, it is easily anticipated that it is hard to acclimate production personnel to invest in professing such intriguing methods. Quality and process control personnel desire simple techniques that they realize the steps taken to reach to a solution. The technique presented here to resolve a fairly complicated situation often encountered in manufacturing operations, that of optimizing process or/and product parameters under multiple specifications concurrently, has several advantages over the aforementioned popular alternatives. One striking advantage is that the use of software is not important with this new method after all. Graphical solutions need not to be drawn. 
TABLE 9: Squared Euclidean norm (sum of weighted squared ranks) for each customer response importance rating.

\begin{tabular}{ccccccccccccccc}
\hline Mn & Mg & Temph & Timeh & AnA & AnB & CR & SSR & SSRA & SSRB & SSRC & SSRD & SSRE & SSRF & MSS \\
\hline 1 & 1 & 1 & 1 & 1 & 1 & 1 & 291.25 & 33.00 & 30.52 & 48.95 & 29.62 & 36.47 & 31.32 & 341 \\
1 & 1 & 1 & 2 & 2 & 2 & 2 & 232.75 & 26.48 & 23.80 & 29.50 & 24.01 & 25.56 & 22.80 & 127 \\
1 & 2 & 2 & 1 & 1 & 2 & 2 & 251.75 & 31.67 & 29.46 & 36.76 & 29.95 & 35.68 & 23.80 & 261 \\
1 & 2 & 2 & 2 & 2 & 1 & 1 & 195.00 & 21.65 & 23.06 & 5.37 & 20.90 & 19.15 & 25.25 & 52 \\
2 & 1 & 2 & 1 & 2 & 1 & 2 & 216.00 & 20.60 & 20.72 & 27.57 & 21.82 & 19.95 & 27.96 & 87 \\
2 & 1 & 2 & 2 & 1 & 2 & 1 & 208.25 & 23.40 & 26.30 & 21.49 & 25.54 & 22.37 & 17.98 & 102 \\
2 & 2 & 1 & 1 & 2 & 2 & 1 & 254.75 & 28.43 & 29.32 & 15.18 & 32.00 & 29.90 & 34.70 & 240 \\
2 & 2 & 1 & 2 & 1 & 1 & 2 & 158.25 & 16.73 & 18.69 & 18.07 & 17.73 & 13.91 & 15.21 & 14 \\
\hline
\end{tabular}

TABLE 10: super-ranking according to each participating customer along with resulting master super-ranking due to simultaneous customer contributions.

\begin{tabular}{ccccccccccccccc}
\hline Mn & Mg & Temph & Timeh & AnA & AnB & CR & SR & SRA & SRB & SRC & SRD & SRE & SRF & MSR \\
\hline 1 & 1 & 1 & 1 & 1 & 1 & 1 & 8 & 8 & 8 & 8 & 6 & 8 & 7 & 8 \\
1 & 1 & 1 & 2 & 2 & 2 & 2 & 5 & 5 & 4 & 6 & 4 & 5 & 3 & 5 \\
1 & 2 & 2 & 1 & 1 & 2 & 2 & 6 & 7 & 7 & 7 & 7 & 7 & 4 & 7 \\
1 & 2 & 2 & 2 & 2 & 1 & 1 & 2 & 3 & 3 & 1 & 2 & 2 & 5 & 2 \\
2 & 1 & 2 & 1 & 2 & 1 & 2 & 4 & 2 & 2 & 5 & 3 & 3 & 6 & 3 \\
2 & 1 & 2 & 2 & 1 & 2 & 1 & 3 & 4 & 5 & 4 & 5 & 4 & 2 & 4 \\
2 & 2 & 1 & 1 & 2 & 2 & 1 & 7 & 6 & 6 & 2 & 8 & 6 & 8 & 6 \\
2 & 2 & 1 & 2 & 1 & 1 & 2 & 1 & 1 & 1 & 3 & 1 & 1 & 1 & 1 \\
\hline
\end{tabular}

Simple calculator work will suffice in producing results while the usage of a spreadsheet may be demanded merely in the most difficult cases. This convenience emanates from the fact that rank statistics are so simple in understanding the meaning of them in the first place and making estimations based on them follows naturally afterwards. This is particularly true for the low sampling schemes this method is intended to be applied at. Suitable OA family candidates will be members of the series $\mathrm{L}_{4}\left(2^{3}\right), \mathrm{L}_{8}\left(2^{7}\right), \mathrm{L}_{12}\left(2^{11}\right)$, and $\mathrm{L}_{16}\left(2^{15}\right)$. In addition, it is easily realizable the extension to nonlinear optimization problems and this will be the subject of a forthcoming publication.

Among the strong points of this extended technique to the one published recently [48] is the convenience in incorporating any number of numerical and categorical responses through the rank-ordering technique. This herein outlined methodology when compared to previous work on the super-ranking concept becomes now a more versatile tool by allowing the inclusion, in a trivial manner, of the weighted influence among the responses according to priorities set by a resourceful customer. Then, in a subsequent, nested super-ranking step, the total behavior elicited by the customer priorities, established by for all considered customers simultaneously, are taken in account in achieving optimal product and process states. The methodology stretches its utility further by implicating in the treatment multiplecustomer priorities that via to the Euclidean norm definition receive a judicious consideration against the engaging production parameters. This is actually accomplished in the second super-ranking step that in principle nests-in the consolidated response information created for each customer separately.
TABle 11: Anderson-Darling Normality Test for the squared Euclidean norm (SSR) shown in Table 3 in the text.

\begin{tabular}{cc}
\hline Levels & Anderson-Darling Normality Test $\mathrm{A}^{2}(P)$ \\
\hline Mn1 & $0.16(.842)$ \\
Mn2 & $0.23(.577)$ \\
Mg1 & $0.40(.176)$ \\
Mg2 & $0.33(.294)$ \\
Temph1 & $0.23(.566)$ \\
Temph2 & $0.29(.384)$ \\
Timeh1 & $0.25(.516)$ \\
Timeh2 & $0.19(.745)$ \\
AnA1 & $0.16(.847)$ \\
AnA2 & $0.15(.873)$ \\
AnB1 & $0.23(.551)$ \\
AnB2 & $0.30(.360)$ \\
CR1 & $0.24(.527)$ \\
CR2 & $0.28(.407)$ \\
\hline
\end{tabular}

Tracking the active effects is of paramount importance even more so often in lean production. Under such circumstances, production teams are often left clueless in lack of systematic and yet convincing solution methods that would otherwise propel them to attain optimal production levels for specifications set simultaneously from a score of different customers without altering their production cycle each time. The case study presented on aluminum milling had that goal in mind to become a useful example for practical implementation in other applications in manufacturing. 

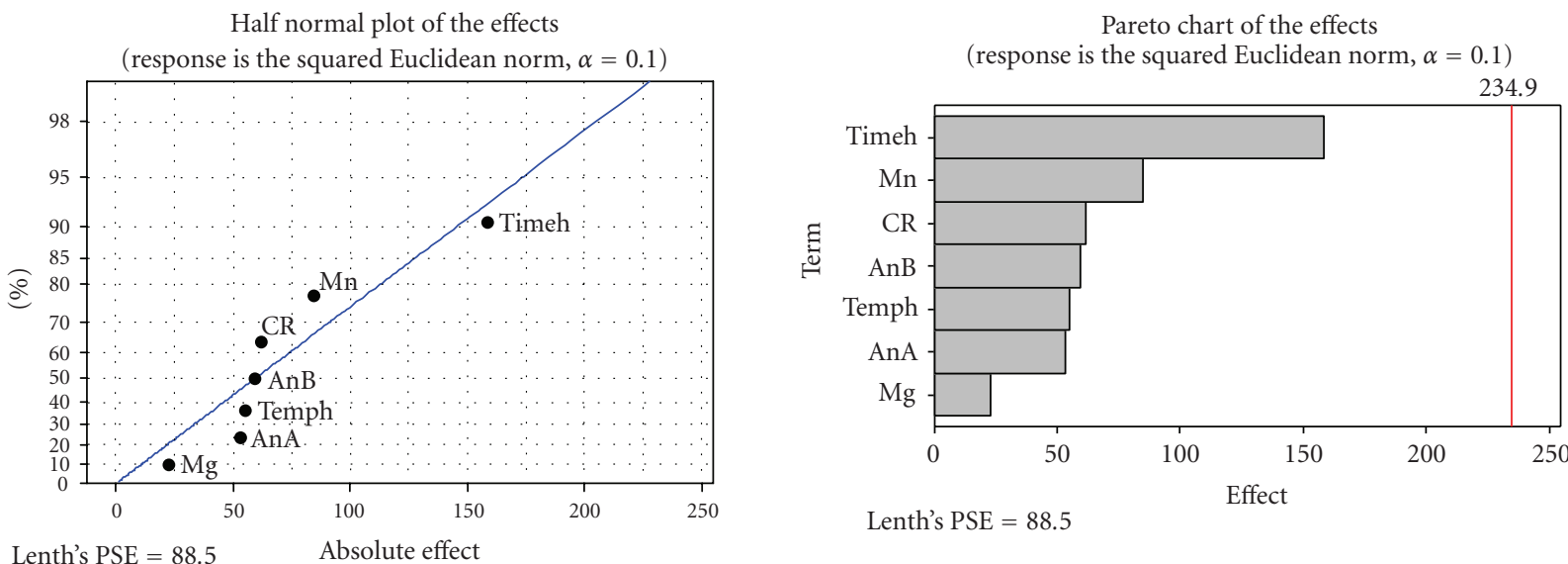

Effect type

- Not Significant

- Significant

(a)

(b)

FIgURE 10: Data for SSR from Table 8 tested with (a) the half-normal test and (b) Pareto analysis/Lenth test.

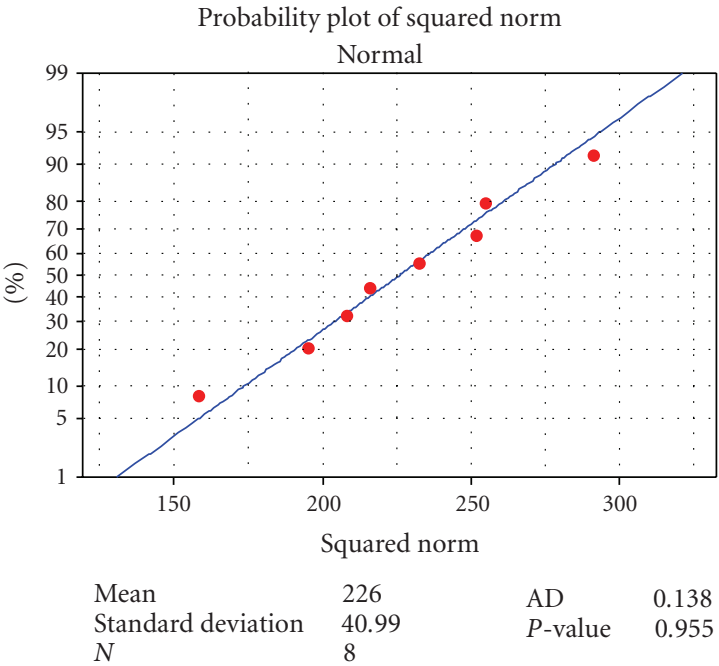

(a)

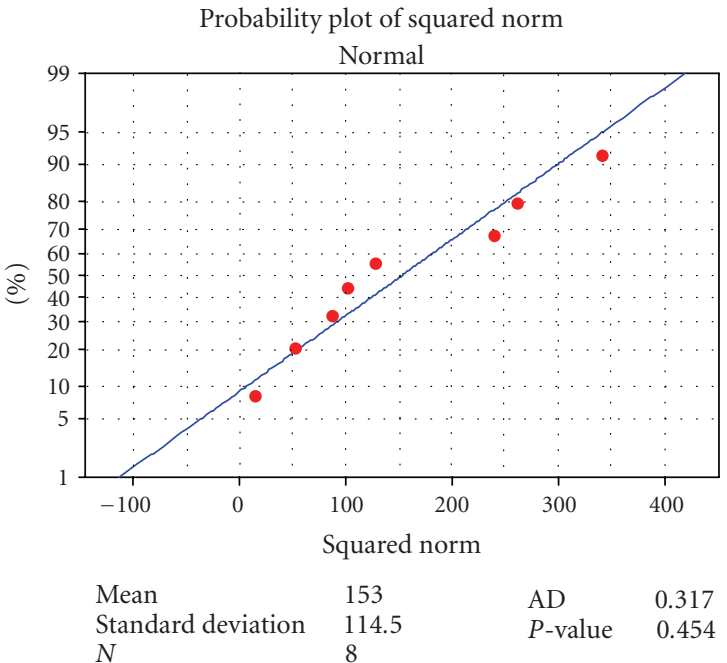

(b)

FIGURE 11: Normality test for SSR values from Table 3 tested (a) and MSS values from Table 9 (b).

The method investigated in this article could have been the sister method for weighted MANOVA. But this latter technique is rendered quickly nullified under the inherent constraint of the unreplication and saturation for fractional factorial schemes imposed invariably on a experimental plan due to anticipated economical requirements and trial duration restrictions that result in calling for exploiting in full OA column occupancy. This nullification stems from the technical complication arising due to the eliminated unexplainable error and the nonexistent degrees of freedom for the error. Therefore, being able to judge statistical significance behind nonrecoverable variability has always been an unwavering challenge with no consensus in sight for a dominating manner of handling it.
The basic assumptions for utilizing the nested superranking concept may be discerned in two parts. The first part has to do with the criteria for selecting a nonparametric tool for multifactorial processing and this has been outlined in detail in [47]. In brief, the main motivation for employing Wilcoxon-Mann-Whitney statistics is the nonreliance on knowing the nature of the distribution of data points within each factor setting. This simplifies greatly the task of analyzing the data when the engineer or scientist that is responsible for carrying out such an endeavour does not possess an in-depth knowledge on statistics. For a typical working sample range of two to six, nonparametrics offer a more objective information imprint comparing to normal statistics as central tendencies based on medians are more 
TABle 12: Anderson-Darling Normality Test for the squared Euclidean norm (MSS) shown in Table 9 in the text.

\begin{tabular}{lc}
\hline Levels & Anderson-Darling Normality Test $\mathrm{A}^{2}(P)$ \\
\hline Mn1 & $0.20(.685)$ \\
Mn2 & $0.29(.404)$ \\
Mg1 & $0.58(.046)$ \\
Mg2 & $0.39(.182)$ \\
Temph1 & $0.16(.847)$ \\
Temph2 & $0.45(.119)$ \\
Timeh1 & $0.27(.439)$ \\
Timeh2 & $0.20(.693)$ \\
AnA1 & $0.21(.637)$ \\
AnA2 & $0.27(.437)$ \\
AnB1 & $0.48(.098)$ \\
AnB2 & $0.36(.244)$ \\
CR1 & $0.23(.584)$ \\
CR2 & $0.22(.616)$ \\
\hline
\end{tabular}

robust in contrast to the means [46]. When factor settings extend to larger sample sizes, benefits gained by the use of order statistics are dissipating unless the distribution of the data is evidently deviant from normality, thus obtaining additional estimations from nonparametrics may be advised. Therefore, the method outlined herein may also complement other leading techniques such as the half-normal plot, the Lenth test, the Bayes plot or the pooled ANOVA approach when there is a discord among their predictions. In as much as there is strong indication that data normality permeates the factor setting differences then any of the aforementioned techniques may be suitable for providing reliable inference. The second part regards the super-ranking concept that it is shown to compete in effectiveness with more elaborate approaches such as a fuzzy logic type of a treatment [48]. super-ranking was shown in the last reference to mix up conveniently and efficiently responses belonging to any of the three categories without requiring particular modification of the ordering transformation tactic. The intrinsic mechanism of forming the superrank vector derives ostensibly but having a theoretical meaning thereof by considering each participating response entry in the Euclidean norm measure.

Another way to treat these problems could have been through the desirability analysis where the importance of each of the responses is encompassed in a model. However, when some of the responses happen to be of categorical nature, like the ones presented in our case study, it is not an easy feat to handle it by this method. Indeed, the incorporation of categorical data was tested in the aluminum product improvement study with limited success. Thus, this is another point where the method of nested superranking presented here may hold an advantage over the desirability method (DM). Rank ordering assimilates easier the inherent variable mixing while establishing statistical significance in the classical sense. One obvious advantage of SR over DM is the way SR establishes a statistical solution irrespectively of some sort of starting values and other peripheral connotations that may need to fuel the DM model. SR produces solutions based on available data hinging on both central tendency and variance effects alike. However, DM operates only on mean estimations that may require further investigation for their statistical significance in the first place. On the other hand, it may be mentioned that DM is driven by connotations rendering each time a new solution.

While the methodology outlined in this paper is recommended to assort the employment of the popular $\mathrm{L}_{4}-\mathrm{L}_{16}$ orthogonal array series, there is no essential restriction for the nonparametric data processing scheme presented in this work to be espoused for any type of two-level fractional factorial design. The interested practitioner merely ought to consult Mann-Whitney's paper for retrieving the appropriate statistical significance limit regarding each time the proper tested sample size.

A great advantage of nested super-ranking is that collapses two kinds of data, responses and their concomitant weights, to a single master response that is expressed by entries belonging to the realms of real numbers. Therefore, the MSS response is easily treated for multicollinearity effects as if it was the classical one-response problem. In turn, formal regression methods may be straightforwardly employed to obtain the estimated regression coefficients $\left(R^{2}\right)$ for every participating factor thus allowing the computation of their tolerances $\left(=1-R^{2}\right)$ leading to the variance inflation factor (VIF). For VIF values greater than 5 the multicollinearity effect should then be detected [49]. Hence, overfitting phenomena may be routinely pinpointed in the proposed method and eliminated.

Another obvious advantage that the nested superranking technique possesses is that by encompassing all information in a new single variable by judiciously absorbing robust representation of data from various sources essentially succeeds in directing the optimum solution according to all product traits simultaneously. This is a highly sought feature in a multiresponse technique that may incorporate multiple correlated responses. When correlated responses are present employing a technique that analyzes each response separately must be avoided, otherwise conflicting recommendations about factor activeness are imminent [50]. Clearly, the suggested method does not ignore a possible correlational nature among the considered responses thus offers objective view of the improvement direction.

Based on the six-customer group (Table 7), the screening optimum factor has been found to be the homogenization duration set at level 2 which corresponds to 24 hours. The confirmation experiment was tested on a cohort of a sixslab batch processing for each customer order separately. Out of the six-slab batch, a three-slab subgroup was randomly selected and their nine product properties were measured and recorded. In a total of eighteen product coils, their properties were analyzed statistically against the predicted product performance. It was found that tensile yield strength, tensile ultimate strength, and sheet thickness were reproducible to their predicted values by an average of $11 \%, 13 \%$, and $7 \%(P<.05)$, respectively. The rest of the product 
traits remained unaffected as predicted by the proposed method.

Lastly, there is always a need to reiterate the advantage the rank statistics maintain over normal-oriented formulations in the low sampling conditions experienced in OA experimentation engaging the family members $\mathrm{L}_{4}\left(2^{3}\right), \mathrm{L}_{8}\left(2^{7}\right)$, $\mathrm{L}_{12}\left(2^{11}\right)$, and $\mathrm{L}_{16}\left(2^{15}\right)$. The superiority in efficiency and the tight confidence intervals that permutation techniques such as the WMW test is known to accomplish provide the necessary and undisputed backbone for robust design through robust statistics. The effect sparsity assumption is abolished just as with the simple SR scheme [47]. Relying on to this method, the philosophical notion about a sample possessing a normal or some other more complicated behavior may not need be of concern for the every day product developer that has to answer pragmatic quality trait puzzles by day's end. Hopefully, this way of data handling as demonstrated here may assist those agonizing industrial practitioners that must provide an answer without feeling apprehended about their lack of an in-depth statistical background or because there is limited 'turn-around' time to place robustness to their decisions.

\section{Conclusion}

A technique has been developed in this paper to assist in resolving unreplicated and saturated multiresponse optimization problems when there is available information about the relative weights among the engaged product characteristics. The technique embodies possible multiple weight assignments for the implicated responses reflecting multicustomer requirements. The nested super-ranking scheme presented here is based on the well-tested nonparametric comparisons while making use of data obtained by an efficient orthogonal array. The massive collapse of multiresponses via order transformation to a single master response grasps multicustomer preferences which in turn are utilized such as production conditions are optimized concurrently for all customers. The theoretical exposition is illustrated by a seven-factor, nine-response, six-customer optimization problem encountered in aluminum milling operations. It is demonstrated that the suggested technique may outperform standard tests of comparisons including the Lenth test and the half-normal plot when some factor setting data may exhibit a tendency towards nonnormal behavior.

\section{Acknowledgments}

The author is greatly indebted to the Editor-in-Chief, Professor Jye-Chyi Lu, and to the Associate Editor, Professor Fugee Tsung, for the efficient handling of this article and their guidance. The careful screening by the two reviewers is recognized that led to constructive comments that aided significantly in the improvement of this work. The author would like to dedicate this effort to the memory of mother, Panagiota, who passed away this summer. She instilled in him the sense of continuous betterment as a principle of life.

\section{References}

[1] D. C. Montgomery, Design and Analysis of Experiments, John Wiley \& Sons, New York, NY, USA, 6th edition, 2004.

[2] C. F. J. Wu and M. Hamada, Experiments: Planning, Analysis and Parameter Design Optimization, John Wiley \& Sons, New York, NY, USA, 2000.

[3] G. E. P. Box, W. G. Hunter, and J. S. Hunter, Statistics for Experimenters: Design, Innovation, Discovery, John Wiley \& Sons, New York, NY, USA, 2nd edition, 2005.

[4] D. Clausing and G. Taguchi, Robust Quality, Harvard Business Review, Tampa, Fla, USA, 1990.

[5] G. Taguchi, Introduction to Quality Engineering, UNIPUB/ Kraus International Publications, White Plains, NY, USA, 1986.

[6] P. J. Ross, Taguchi Techniques for Quality Engineering, McGraw-Hill, New York, NY, USA, 1988.

[7] E. A. Elsayed and A. Chen, "Optimal levels of process parameters for products with multiple characteristics," International Journal of Production Research, vol. 31, no. 5, pp. 1117-1132, 1993.

[8] G. G. Vining and R. H. Myers, "Combining Taguchi and response surface philosophies: a dual response approach," Journal of Quality Technology, vol. 22, no. 1, pp. 38-45, 1990.

[9] L. C. Tang and K. Xu, "A unified approach for dual response surface optimization," Journal of Quality Technology, vol. 34, no. 4, pp. 437-447, 2002.

[10] G. Box, "Signal-to-noise ratios, performance criteria, and transformations," Technometrics, vol. 30, no. 1, pp. 1-17, 1988.

[11] R. H. Myers, D. C. Montgomery, G. G. Vining, C. M. Borror, and S. M. Kowalski, "Response surface methodology: a retrospective and literature survey," Journal of Quality Technology, vol. 36, no. 1, pp. 53-78, 2004.

[12] T. J. Robinson, C. M. Borror, and R. H. Myers, "Robust parameter design: a review," Quality and Reliability Engineering International, vol. 20, no. 1, pp. 81-101, 2004.

[13] A. I. Khuri and M. Conlon, "Simultaneous optimization of multiple responses represented by polynomial regression functions," Technometrics, vol. 23, no. 4, pp. 363-375, 1981.

[14] J. Tao, A. J. Shih, and J. Ni, "Experimental study of the dry and near-dry electrical discharge milling processes," Journal of Manufacturing Science and Engineering, vol. 130, no. 1, Article ID 011002, 9 pages, 2008.

[15] J. P. Jordaan and C. P. Ungerer, "Optimization of design tolerances through response surface approximations," Journal of Manufacturing Science and Engineering, vol. 124, no. 3, pp. 762-767, 2002.

[16] J. S. Chung and S. M. Hwang, "Process optimal design in forging by genetic algorithm," Journal of Manufacturing Science and Engineering, vol. 124, no. 2, pp. 397-408, 2002.

[17] A. Gupta, Y. Ding, L. Xu, and T. Reinikainen, "Optimal parameter selection for electronic packaging using sequential computer simulations," Journal of Manufacturing Science and Engineering, vol. 128, no. 3, pp. 705-715, 2006.

[18] C.-X. Feng and A. Kusiak, "Robust tolerance synthesis with the design of experiments approach," Journal of Manufacturing Science and Engineering, vol. 122, no. 3, pp. 520-528, 2000.

[19] J. A. Harding, M. Shahbaz, Srinivas, and A. Kusiak, "Data mining in manufacturing: a review," Journal of Manufacturing Science and Engineering, vol. 128, no. 4, pp. 969-976, 2006.

[20] J. J. Pignatiello, "Strategies for robust multiresponse quality engineering," IIE Transactions, vol. 25, no. 3, pp. 5-15, 1993. 
[21] D. K. Sobek, A. C. Ward, and J. K. Liker, "Toyota's principles of set-based concurrent engineering," Sloan Management Review, vol. 40, no. 2, pp. 67-83, 1999.

[22] Y.-E. Nahm, H. Ishikawa, and Y.-S. Yang, "A flexible and robust approach for preliminary engineering design based on designer's preference," Concurrent Engineering Research and Applications, vol. 15, no. 1, pp. 53-62, 2007.

[23] P. Das, "Concurrent optimization of multiresponse product performance," Quality Engineering, vol. 11, no. 3, pp. 365-368, 1999.

[24] G. Derringer and R. Suich, "Simultaneous optimization of several response variables," Journal of Quality Technology, vol. 12, no. 4, pp. 214-219, 1980.

[25] E. Del Castillo, D. C. Montgomery, and D. R. McCarville, "Modified desirability functions for multiple response optimization," Journal of Quality Technology, vol. 28, no. 3, pp. 337-345, 1996.

[26] P. Goik, J. W. Liddy, and W. Taam, "Use of desirability functions to determine operating windows for new product designs," Quality Engineering, vol. 7, no. 2, pp. 267-276, 1994.

[27] C. Ribardo and T. T. Allen, "An alternative desirability function for achieving 'six sigma' quality," Quality and Reliability Engineering International, vol. 19, no. 3, pp. 227-240, 2003.

[28] F. Ortiz Jr., J. R. Simpson, J. J. Pignatiello Jr., and A. HerediaLangner, "A genetic algorithm approach to multiple-response optimization," Journal of Quality Technology, vol. 36, no. 4, pp. 432-450, 2004.

[29] F.-C. Wu, "Optimization of correlated multiple quality characteristics using desirability function," Quality Engineering, vol. 17, no. 1, pp. 119-126, 2005.

[30] K.-J. Kim and D. K. J. Lin, "Simultaneous optimization of mechanical properties of steel by maximizing exponential desirability functions," Journal of the Royal Statistical Society: Series C, vol. 49, no. 3, pp. 311-325, 2000.

[31] J. F. Kros and C. M. Mastrangelo, "Comparing multi-response design methods with mixed responses," Quality and Reliability Engineering International, vol. 20, no. 5, pp. 527-539, 2004.

[32] R. C. Wurl and S. L. Albin, "A comparison of multiresponse optimization: sensitivity to parameter selection," Quality Engineering, vol. 11, no. 3, pp. 405-415, 1999.

[33] A. E. Ames, N. Mattucci, S. MacDonald, G. Szonyi, and D. M. Hawkins, "Quality loss functions for optimization across multiple response surfaces," Journal of Quality Technology, vol. 29, no. 3, pp. 339-346, 1997.

[34] G. G. Vining, "A compromise approach to multiresponse optimization," Journal of Quality Technology, vol. 30, no. 4, pp. 309-313, 1998.

[35] D. Romano, M. Varetto, and G. Vicario, "Multiresponse robust design: a general framework based on combined array," Journal of Quality Technology, vol. 36, no. 1, pp. 27-37, 2004.

[36] R. Berni and C. Gonnelli, "Planning and optimization of a numerical control machine in a multiple response case," Quality and Reliability Engineering International, vol. 22, no. 5, pp. 517-526, 2006.

[37] P. W. Phillips and K.-J. Kim, "Taguchi parameter design with multiple quality characteristics," Quality Management Journal, vol. 6, no. 4, pp. 26-40, 1999.

[38] P. B. S. Reddy, K. Nishina, and A. S. Babu, "Unification of robust design and goal programming for multiresponse optimization-a case study," Quality and Reliability Engineering International, vol. 13, no. 6, pp. 371-383, 1997.

[39] L.-I. Tong and K.-L. Hsieh, "A novel means of applying neural networks to optimize the multiresponse problem," Quality Engineering, vol. 13, no. 1, pp. 11-18, 2000.
[40] K.-L. Hsieh and L.-I. Tong, "Optimization of multiple quality responses involving qualitative and quantitative characteristics in IC manufacturing using neural networks," Computers in Industry, vol. 46, no. 1, pp. 1-12, 2001.

[41] P. Kumar, P. B. Barua, and J. L. Gaindhar, "Quality optimization (multi-characteristics) through Taguchi's technique and utility concept," Quality and Reliability Engineering International, vol. 16, no. 6, pp. 475-485, 2000.

[42] J. Antony, "Multi-response optimization in industrial experiments using Taguchi's quality loss function and principal component analysis," Quality and Reliability Engineering International, vol. 16, no. 1, pp. 3-8, 2000.

[43] L.-I. Tong and C.-H. Wang, "Multi-response optimization using principal component analysis and grey relational analysis," International Journal of Industrial Engineering, vol. 9, no. 4, pp. 343-350, 2002.

[44] W. M. Carlyle, D. C. Montgomery, and G. C. Runger, "Optimization problems and methods in quality control and improvement," Journal of Quality Technology, vol. 32, no. 1, pp. $1-17,2000$.

[45] C. K. Ch'ng, S. H. Quah, and H. C. Low, "A new approach for multiple-response optimization," Quality Engineering, vol. 17, no. 4, pp. 621-626, 2005.

[46] W. J. Conover, Practical Nonparametric Statistics, Academic Internet, New York, NY, USA, 3rd edition, 2006.

[47] G. J. Besseris, "Analysis of an unreplicated fractional-factorial design using nonparametric tests," Quality Engineering, vol. 20, no. 1, pp. 96-112, 2008.

[48] G. J. Besseris, "Multi-response optimisation using Taguchi method and super ranking concept," Journal of Manufacturing Technology Management, vol. 19, no. 8, pp. 1015-1029, 2008.

[49] R. M. O'Brien, "A caution regarding rules of thumb for variance inflation factors," Quality \& Quantity, vol. 41, no. 5, pp. 673-690, 2007.

[50] C.-H. Chiao and M. Hamada, "Analyzing experiments with correlated multiple responses," Journal of Quality Technology, vol. 33, no. 4, pp. 451-465, 2001. 

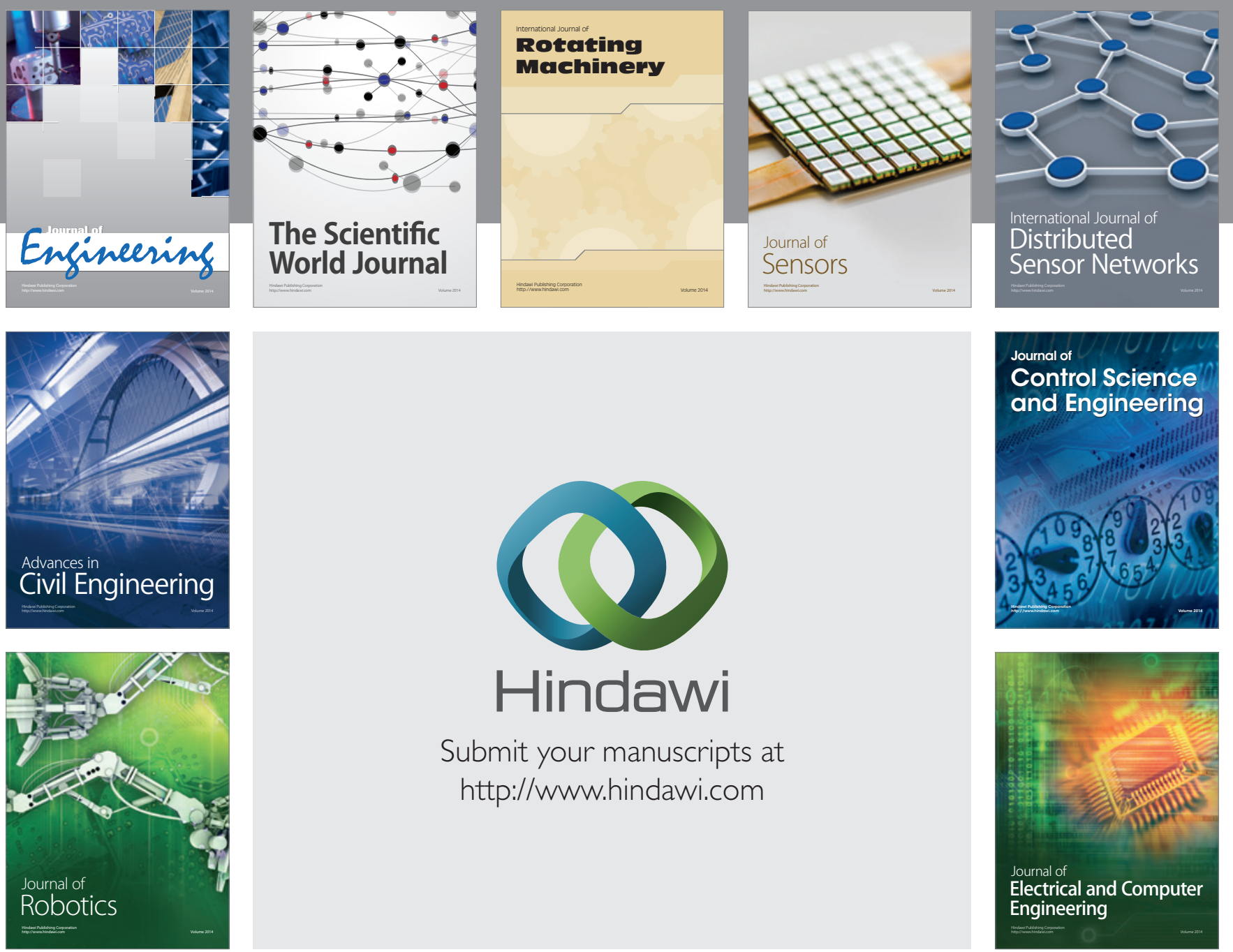

Submit your manuscripts at

http://www.hindawi.com
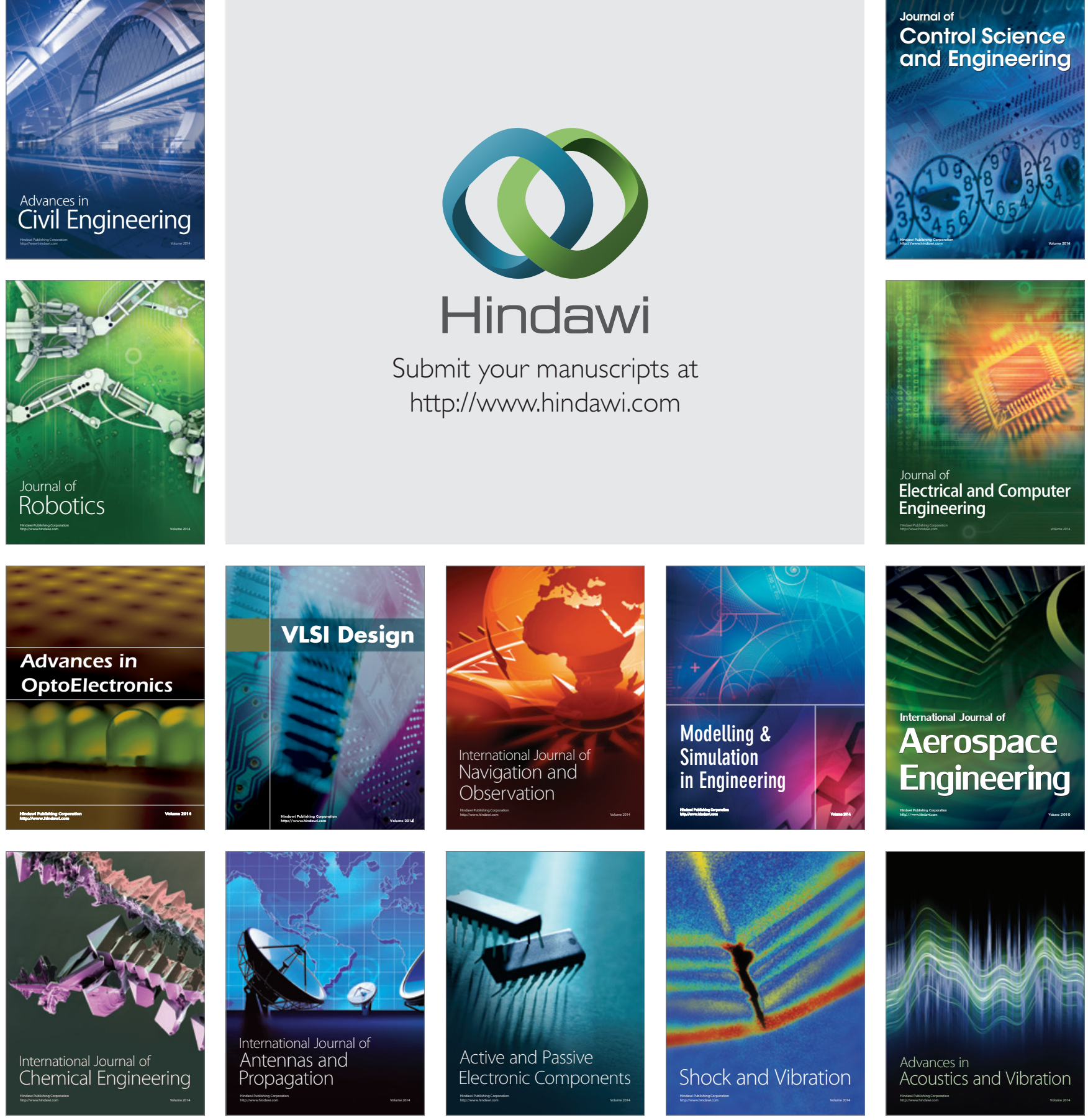